\title{
Evaluation of MGIT 960 System for the Second-Line Drugs Susceptibility Testing of Mycobacterium tuberculosis
}

\author{
Hyejin Kim, ${ }^{1}$ Minji Seo, ${ }^{2}$ Young Kil Park, ${ }^{1}$ Jae-Il Yoo, ${ }^{3}$ Yeong Seon Lee, ${ }^{3}$ \\ Gyung Tae Chung, ${ }^{3}$ and Sungweon Ryoo ${ }^{1}$ \\ ${ }^{1}$ Korean Institute of Tuberculosis, 168-5, Osong sangmyung 4 ro, Osong-eup, Cheongwon-gun, Chungbuk 363-954, Republic of Korea \\ ${ }^{2}$ Novartis Korea LTD, CD\&MA, Namdaemunro 5-ga, Joon-gu 100-753, Republic of Korea \\ ${ }^{3}$ Centers for Infectious Diseases, National Institute of Health, Korea Centers for Disease Control and Prevention, \\ 187 Osong sangmyung 2 ro, Osong-eup, Cheongwon-gun, Chungbuk 363-951, Republic of Korea
}

Correspondence should be addressed to Sungweon Ryoo; scientist1@empal.com

Received 24 December 2012; Revised 7 March 2013; Accepted 11 March 2013

Academic Editor: Alexander S. Apt

Copyright (C) 2013 Hyejin Kim et al. This is an open access article distributed under the Creative Commons Attribution License, which permits unrestricted use, distribution, and reproduction in any medium, provided the original work is properly cited.

\begin{abstract}
Many laboratories validate DST of the second-line drugs by BACTEC MGIT 960 system. The objective of this study is to evaluate the critical concentration and perform DST for the 2nd line drugs. We evaluated 193 clinical strains of $M$. tuberculosis isolated from patients in South Korea. Testing the critical concentration of six second-line drugs was performed by MGIT 960 and compared with L-J proportion method. The critical concentration was determined to establish the most one that gave the difference between drug resistance and susceptibility in MGIT960 system. Good agreement of the following concentrations was found: Concordance was $95 \%$ for $0.5 \mu \mathrm{g} / \mathrm{mL}$ of moxifloxacin; $93.6 \%, 1.0 \mu \mathrm{g} / \mathrm{mL}$ of levofloxacin; $97.5 \%, 2.5 \mu \mathrm{g} / \mathrm{mL}$ of kanamycin; $90.6 \%, 2.5 \mu \mathrm{g} / \mathrm{mL}$ of capreomycin; $86.2 \%, 5.0 \mu \mathrm{g} / \mathrm{mL}$ of ethionamide; and $90.8 \%, 2.0 \mu \mathrm{g} / \mathrm{mL}$ of $\rho$-aminosalicylic acid. The critical concentrations of the four drugs, moxifloxacin, levofloxacin, kanamycin, and capreomycin, were concordant and reliable for testing 2 nd line drug resistance. Further study of ethionamide and $\rho$-aminosalicylic acid is required.
\end{abstract}

\section{Introduction}

Tuberculosis represents a major public health concern especially due to the increasing number of multidrug-resistant tuberculosis TB (MDR TB). Particularly in developing countries, extensively drug-resistant tuberculosis TB (XDR TB) continues to pose serious problem [1-5]. The increase in MDR/XDR TB rates prompts effective diagnostic methods so that appropriate treatments can be given to infected patients [6-8]. Many studies reported that MGIT 960 (Becton Dickinson Diagnostic System, Sparks, MD) provided reliable and rapid results in the detection and recovery of mycobacterium from clinical specimens and also the drug susceptibility testing (DST) of the TB isolates for the first line drugs isoniazid, rifampicin, ethambutol, streptomycin, and pyrazinamide [9, 10]. Recently, laboratories are facing great hindrances to provide DST for second-line drugs to ensure effective treatment of MDR/XDR TB by using MGIT 960 system [1116]. However, in most of these studies, the tested numbers of second-line anti-TB drug were limited and the critical concentrations ranges of the second-line drugs were also discordant [11-13]. Despite the recommendation by WHO in 2008 for the use of liquid media for the second-line DST using MGIT 960, it is still unreliable due to the difficulty in determining the critical concentration [16]. It is not easy to calibrate newly developed DST methods using altered conditions. In vitro results of DST to second-line drugs were affected by criteria for measuring resistance such as the critical concentrations and critical proportions of drugs. For instance, DST with L-J solid media was established MICs that was defined as the drug concentration on which $<20$ colonies were found, while MIC in MGIT 960 was defined as the drug concentration at which the daily change in growth unit was less than that of the 1:100 control [17].

The Korean Institute of Tuberculosis (KIT) is a government operating research institute founded in 1970. KIT has been working as a main organization for conducting National TB program including diagnosis, basic research, and vaccine 
production. According to Korean Guidelines for Tuberculosis there are 5 practical tips for building treatment regimen for MDR-TB patients. Step 1 includes available 1st line drugs: pyrazinamide and ethambutol. Step 2 : choose one of the injectable drugs: kanamycin, amikacin, streptomycin, capreomycin. Step 3: choose one of the quinolone drugs: levofloxacin, moxifloxacin, ofloxacin. Step 4: choose available drugs among group IV: $\rho$-aminosalicylic acid, cycloserine, prothionamide. Step 5: if 4 drugs are not composed until step 4 group V drugs can be considered: amoxicillin/clavulanate, clarithromycin, high-dose isoniazid, clofazimine, linezolid, thioacetazone (not for HIV patients).

The L-J proportion method has been used for both firstand second-line DST for a long time in Korea, but recently, a switch to MGIT 960 method has been initiated for firstline DST. In this study, we tested the various critical concentrations published second-line TB drugs, moxifloxacin, levofloxacin, kanamycin, capreomycin, ethionamide, and $\rho$ aminosalicylic acid and used L-J proportion method as a reference for comparison with the MGIT 960 system.

\section{Materials and Methods}

2.1. Strains. Cultures submitted to the Korean Institute of Tuberculosis from the Public Health Center in Korea were evaluated. A total of 193 clinical strains of M. tuberculosis, including 134 pansusceptible strains and 59 resistant strains whose drug susceptibility results were previously determined by L-J, were tested. All isolates were analyzed by each patient category with treatment history (Table 1). All isolates were freshly subcultured on L-J medium before being used and tested by the MGIT 960.

2.2. Chemicals. All drugs were in chemically pure powder form. Levofloxacin (LEV), Capreomycin (CPM), Kanamycin (KM), Ethionamide (ETH), and $\rho$-aminosalicylic acid (PAS) were obtained from Sigma-Aldrich (St. Louis, MO), and Moxifloxacin (MXF) was obtained from Bayer (Bayer Health Care AG, Germany). The powders were stored at $-20^{\circ} \mathrm{C}$ in desiccators as recommended by the manufacturer. LEV, $\mathrm{CPM}, \mathrm{KM}$, and PAS were dissolved in deionized water (DW), MXF in $0.1 \mathrm{~N} \mathrm{NaOH}$ with subsequent dilutions in DW, and ETH solubilized in DMSO with subsequent dilutions in DW. All stock solutions were sterilized by membrane filtration through $0.22 \mu \mathrm{m}$-pore-size Millex-GS filter units (Millipore, Bedford, MA). All stock solutions were stored at $-80^{\circ} \mathrm{C}$ in small aliquots. The frozen drug solutions were used immediately after thawing and the remaining was discarded and never stored in freezer again. Working solution was prepared freshly from the stock solution and the serial dilutions were carried out to achieve the desired concentrations.

2.3. Drug Susceptibility Test by the MGIT960 Method. MGIT DST was performed with strains grown from L-J slope, which were identified as $M$. tuberculosis with $\mathrm{ZN}$ staining. For the preparation of inoculums from L-J culture, $4 \mathrm{~mL}$ of Middlebrook 7H9 broth was added to a sterile tube with cap containing six to ten $2 \mathrm{~mm}$ glass beads. 1 2 colonies were
TABLE 1: Clinical M. tuberculosis strains used in the study.

\begin{tabular}{lcc}
\hline \multirow{2}{*}{ Test drugs } & \multicolumn{2}{c}{ Number of strains } \\
& Susceptible & Resistant $^{\mathrm{a}}$ \\
\hline MXF & 37 & 4 \\
$\quad$ New cases & 3 & 15 \\
$\quad$ Previously treated cases & 40 & \\
LEV & 0 & 4 \\
$\quad$ New cases & & 15 \\
$\quad$ Previously treated cases & 40 & 6 \\
KM & 0 & 10 \\
$\quad$ New cases & 39 & \\
$\quad$ Previously treated cases & 1 & 5 \\
CPM & & 6 \\
$\quad$ New cases & 39 & 2 \\
Previously treated cases & 0 & 6 \\
ETH & & 6 \\
New cases & 40 & 11 \\
Previously treated cases & 0 & \\
PAS & & \\
New cases & & \\
Previously treated cases &
\end{tabular}

${ }^{\mathrm{a}}$ Treatment period of drug in resistant strains was $>6$ months.

extracted using a sterile loop from a growth culture that was not older than 14 days. The colonies were suspended in the Middlebrook 7H9 broth and vortexed for 2-3 minutes to break up the larger clumps. The suspension was left for 30 minutes to settle down the sediment to the bottom of the tube. Then, the supernatant fluid was transferred to another sterile tube and the suspension was left for another $15 \mathrm{~min}$. Using 7H9 broth, the suspension was adjusted to a 0.5 McFarland standard. $1 \mathrm{~mL}$ of the adjusted suspension was diluted in $4 \mathrm{~mL}$ of sterile saline (1:5 dilutions). A $0.5 \mathrm{~mL}$ of the $1: 5$ dilutions was inoculated into tubes containing test drugs. In preparation of the second-line drug growth control tube, $0.1 \mathrm{~mL}$ of the previously mentioned suspension was pipetted into a total of $10 \mathrm{~mL}$ of sterile saline to prepare the 1:100 GC suspensions (1\% growth control). The GC suspension was mixed thoroughly by gently inverting 3 to 4 times and then inoculated with $0.5 \mathrm{~mL}$ of the $1: 100 \mathrm{GC}$ suspensions into MGIT tubes. To interpret the results for 2nd drugs, the standard protocol recommended by the first-line drug manufacturers was followed for DST by the MGIT 960 method. When the growth unit (GU) of the growth control reaches 400 within 4-13 days, the GUs values of the drug-containing vials were evaluated. The GU of the drug-containing tubes were found 100 and $>100$; the result reported susceptible and resistant strains, respectively. Evaluated concentrations of each drug are listed in Table 2.

2.4. Drug Susceptibility Test by the L-J Proportion Method. L-J proportion method was the reference method for this study. All strains were previously tested on L-J medium by standard procedures at concentrations of MXF $2.0 \mu \mathrm{g} / \mathrm{mL}, \mathrm{LEV}$ 
TABLE 2: Drug concentrations tested in MGIT 960 and L-J proportion method.

\begin{tabular}{lcc}
\hline \multirow{2}{*}{ Drug } & \multicolumn{2}{c}{ Concentration evaluated $(\mu \mathrm{g} / \mathrm{mL})$} \\
& MGIT960 & L-J \\
\hline Moxifloxacin & $0.25,0.5,1.0,2.0,4.0$ & 2.0 \\
Levofloxacin & $0.25,0.5,1.0,2.0,4.0,8.0$ & 2.0 \\
Kanamycin & $0.625,1.25,2.5,5.0,6.0,10.0$ & 40.0 \\
Capreomycin & $0.625,1.25,2.5,5.0$ & 40.0 \\
Ethionamide & $0.625,1.25,2.5,5.0$ & 40.0 \\
$\rho$-aminosalicylic acid & $0.5,1.0,2.0,4.0,8.0,16.0$ & 1.0 \\
\hline
\end{tabular}

$2.0 \mu \mathrm{g} / \mathrm{mL}, \mathrm{KM} 40 \mu \mathrm{g} / \mathrm{mL}$, CPM $40 \mu \mathrm{g} / \mathrm{mL}$, ETH $40 \mu \mathrm{g} / \mathrm{mL}$, and PAS $1.0 \mu \mathrm{g} / \mathrm{mL}[18]$.

2.5. Genotypic Characterization. Strains that have shown discrepancy between the MGIT960 and L-J DST were retested in two methods. Also, we analyzed by DNA sequencing the key regions involved in the following genes; gyrA and $g y r B$ for MXF and LEV, rrs for both KM and CPM, tlyA for CPM, and inhA promoter, inhA, ethA, and ethR for ETH.

\section{Results and Discussion}

A total of 193 strains including 134 pansusceptible strains and 59 resistant strains with various drug susceptibility profiles (19 isolates were resistant to MXF and LEV, 16 isolates were resistant to $\mathrm{KM}, 11$ isolates were resistant to $\mathrm{CPM}, 8$ isolates were resistant to ETH, and 17 isolates were resistant to PAS according to the L-J method) were tested for susceptibilities for the second-line drugs. To determine the minimal inhibitory antimicrobial test concentrations (MICs) for 6 drugs, we analyzed twelve references having DST data using MGIT 960 for second-line drugs. To determine the critical concentration, it is based on the basis of distinguishable susceptible and resistant strains. The DST results for each 6 drugs are presented in Table 3 . On testing both susceptible and resistant strains, the critical concentrations were obtained: $0.5 \mu \mathrm{g} / \mathrm{mL}$ for MXF, $1.0 \mu \mathrm{g} / \mathrm{mL}$ for $\mathrm{LEV}, 2.5 \mu \mathrm{g} / \mathrm{mL}$ for $\mathrm{KM}$, $2.5 \mu \mathrm{g} / \mathrm{mL}$ for CPM, $5.0 \mu \mathrm{g} / \mathrm{mL}$ for ETH, and $2.0 \mu \mathrm{g} / \mathrm{mL}$ for PAS. Good agreement of critical concentration between the MGIT 960 and L-J proportion method results was observed for MXF, LEV, KM, and CPM.

The MICs for MXF have been reported between 0.125 and $2.0 \mu \mathrm{g} / \mathrm{mL}$ and resistant strains for this drug are characterized by a higher MIC $[12,13,19,20]$. For example, MIC of clinical isolates and pansusceptible strains was reported on the basis of the MGIT960 method [12]. Also, it was reported that MICs of gyrA mutant strains were higher than those for pansusceptible and resistant strains [21]. We evaluated extended concentrations among $0.25 \sim 4.0 \mu \mathrm{g} / \mathrm{mL}$ to determine MIC of resistant strain for MXF in this study (Table 2).

For moxifloxacin, the agreement was $95 \%$ concordant at $0.5 \mu \mathrm{g} / \mathrm{mL}$. We found four strains with discordant result. One of those isolates was resistant at $1.0 \mu \mathrm{g} / \mathrm{mL}$ as false resistant when repeated using the MGIT960 method. Three strains were susceptible when tested by L-J method, but resistant (at $<0.5 \mu \mathrm{g} / \mathrm{mL}$ ) and susceptible (at $\geq 1.0 \mu \mathrm{g} / \mathrm{mL}$ ) when tested by MGIT960 (Table 4). Historically, those isolates were from each patient in the followup. To elucidate isolates with reduced susceptibility to MXF, we analyzed the mutation associated with resistance to MXF. Although either gyrA or gyrB mutations was not detected, those strains possibly carry a mutation which is located outside the QRDRs, or the resistance may be caused by other mechanisms.

The MICs for LEV were recommended between 0.5 and $2.0 \mu \mathrm{g} / \mathrm{mL}$ in previous papers [22, 23]. According to Sanders's paper, they tested MICs with both pansusceptible and resistant strain. MICs of resistant strains $(2.0 \mu \mathrm{g} / \mathrm{mL})$ were higher than those for the pansusceptible strains $(1.0 \mu \mathrm{g} / \mathrm{mL})$, which showed bimodal pattern like MXF [24]. We evaluated concentrations among $0.25 \sim 8.0 \mu \mathrm{g} / \mathrm{mL}$ for LEV to determine MIC of resistant strains (Table 2). For levofloxacin, the agreement was $94 \%$ at $1.0 \mu \mathrm{g} / \mathrm{mL}$. Applying the $1.0 \mu \mathrm{g} / \mathrm{mL}$ for the MGIT method, 18 of 19 resistant strains yielded resistant results and 37 of 40 susceptible strains yielded susceptible results (sensitivity $94.7 \%$, specificity $92.5 \%$ ). The three susceptible strains were susceptible at $>1.0 \mu \mathrm{g} / \mathrm{mL}$ when tested using the MGIT960 method. For the three susceptible strains, we analyzed gene sequence in those discordant strains and did not harbor the gyrA and $g y r B$ mutations (Table 4).

The MICs for KM were recommended $1.25 \sim 4.0 \mu \mathrm{g} / \mathrm{mL}$ in the previous reports $[19,20]$. Critical concentration of this drug is commonly recommended between 2.5 and $5.0 \mu \mathrm{g} / \mathrm{mL}$ but it is greater than $20 \mu \mathrm{g} / \mathrm{mL}$ in the case of using KMresistant strains $[6,11,19]$. Range of MIC was chosen 0.625 $10 \mu \mathrm{g} / \mathrm{mL}$ to evaluate MICs of KM in our study (Table 2).

For kanamycin, the use of the $2.5 \mu \mathrm{g} / \mathrm{mL}$ critical concentration for the MGIT960 method led to yielding 97.5\% concordance. 16 of 16 resistant strains yielded resistant results and 38 of 40 susceptible strains yielded susceptible results. The two discrepant strains did not harbor the rrs mutation (Table 4). We suggest the critical concentration for $\mathrm{KM}$ as a $2.5 \mu \mathrm{g} / \mathrm{mL}$ to yield the best discrimination between susceptible and resistant strains.

The MICs for CPM were reported 1.0 5.0 $\mu \mathrm{g} / \mathrm{mL}[19,23$, 25]. We evaluated MICs of CPM among $0.625 \sim 5.0 \mu \mathrm{g} / \mathrm{mL}$ (Table 2). The concordance between both DST methods was $91 \%$ in capreomycin testing. Applying the $2.5 \mu \mathrm{g} / \mathrm{mL}$ for the MGIT method, 10 of 11 resistant strains yielded resistant results and 37 of 40 susceptible strains yielded susceptible results (sensitivity $90.9 \%$, specificity $90.3 \%$ ). Three discordant susceptible strains were susceptible at $>2.5 \mu \mathrm{g} / \mathrm{mL}$ when tested using the MGIT960 method. Those strains were sequenced $r r s$ and tly $A$ genes for genetic analysis but no mutations were detected in the two genes (Table 4). Resistant strain was found to be susceptible at $>0.625 \mu \mathrm{g} / \mathrm{mL}$ by MGIT 960 . This isolate was from XDR-TB patient in the 4th follow-up sample. This strain also proved susceptible at $>0.625 \mu \mathrm{g} / \mathrm{mL}$ by MGIT 960 but resistant by L-J method. The resistance to fluoroquinolones was reported; aminoglycosides were developed during therapy [26]. Although it was isolated from patient during treatment, it seemed to obtain more susceptible results with the MGIT 960 instrumentation than with the comparative L-J method. 
TABLE 3: Drug susceptibility testing results of each drug determined by use of the MGIT 960 system to be compared with L-J.

\begin{tabular}{|c|c|c|c|c|c|c|c|}
\hline \multirow{2}{*}{ Drug } & \multirow{2}{*}{ Conc. evaluated $(\mu \mathrm{g} / \mathrm{mL})$} & \multicolumn{4}{|c|}{ No. of strains with indicated results by L-J/MGIT 960} & \multirow{2}{*}{ Sensitivity (\%) } & \multirow{2}{*}{ Specificity (\%) } \\
\hline & & $R / R$ & $S / S$ & $R / S$ & $S / R$ & & \\
\hline MXF & 0.5 & 19 & 36 & 0 & 4 & 100 & 90.0 \\
\hline LEV & 1.0 & 18 & 37 & 1 & 3 & 94.7 & 92.5 \\
\hline KM & 2.5 & 16 & 38 & 0 & 2 & 100 & 95.0 \\
\hline CPM & 2.5 & 10 & 37 & 1 & 3 & 90.9 & 90.3 \\
\hline ETH & 5.0 & 6 & 38 & 2 & 1 & 75.0 & 97.4 \\
\hline PAS & 2.0 & 16 & 35 & 1 & 5 & 94.1 & 87.5 \\
\hline
\end{tabular}

TABLE 4: Drug susceptibility and genotypic characterization of isolates show discrepant results between L-J and Bactec MGIT 960.

\begin{tabular}{|c|c|c|c|c|c|c|}
\hline Discordant isolates & \multicolumn{4}{|c|}{ Susceptibility at the following concentration $(\mu \mathrm{g} / \mathrm{mL})$} & \multirow[t]{2}{*}{ KIT number } & \multirow[t]{2}{*}{ Genotypic characterization } \\
\hline MXF & 0.25 & 0.5 & 1.0 & & & \\
\hline \multirow{2}{*}{$S / R$} & $R$ & $R$ & $S$ & & $1176,1503,1574$ & gyrA, wt; gyrB, wt \\
\hline & $R$ & $R$ & $R$ & & $1575^{\mathrm{a}}$ & \\
\hline LEV & 0.5 & 1.0 & 2.0 & & & \multirow{3}{*}{ gyrA, wt; gyrB, wt } \\
\hline$R / S$ & $R$ & $S$ & $S$ & & $2933^{\mathrm{b}}$ & \\
\hline$S / R$ & $R$ & $R$ & $S$ & & $1147,1155,4900$ & \\
\hline KM & 1.25 & 2.5 & 5.0 & & & \\
\hline$S / R$ & $R$ & $R$ & $R$ & & 1562,1565 & $r r s, \mathrm{wt}$ \\
\hline CPM & 0.625 & 1.25 & 2.5 & 5.0 & & \\
\hline$R / S$ & $R$ & $S$ & $S$ & $S$ & 4831 & rrs, wt; tlyA, wt \\
\hline$S / R$ & $R$ & $R$ & $R$ & $S$ & $1168,1182,2809$ & \\
\hline ETH & 1.25 & 2.5 & 5.0 & & & \multirow{4}{*}{$\begin{array}{c}\text { eth } A, \text { ethR, inh } A, \text { wt } \\
\text { eth } A, \text { S266R; ethR, inhA, wt } \\
\text { eth } A, \text { ethR, inh } A, w t\end{array}$} \\
\hline \multirow{2}{*}{$R / S$} & $R$ & $S$ & $S$ & & 5189 & \\
\hline & $R$ & $R$ & $S$ & & $5298^{c}$ & \\
\hline$S / R$ & $R$ & $R$ & $R$ & & 2934 & \\
\hline PAS & 0.5 & 1.0 & 2.0 & 4.0 & & \multirow{4}{*}{ Not done } \\
\hline$R / S$ & $R$ & $S$ & $S$ & $S$ & 4891 & \\
\hline \multirow{2}{*}{$S / R$} & $R$ & $R$ & $R$ & $S$ & $1201,1212,1214,1573$ & \\
\hline & $\mathrm{R}$ & $\mathrm{R}$ & $\mathrm{R}$ & $\mathrm{R}$ & 4537 & \\
\hline
\end{tabular}

${ }^{\mathrm{a}}$ Determined as a false-resistant result.

${ }^{\mathrm{b}}$ Treatment period of drug in this patient was 17 months.

${ }^{\mathrm{c}}$ Determined as a false-susceptible result.

ETH was recommended among $0.5 \sim 2.5 \mu \mathrm{g} / \mathrm{mL}[19,23$, $25,27]$. We evaluated among $0.625 \sim 5.0 \mu \mathrm{g} / \mathrm{mL}$ for $\mathrm{ETH}$ (Table 2).

For ethionamide, we proposed $5.0 \mu \mathrm{g} / \mathrm{mL}$ critical concentrations in the MGIT960. Using a concentration of $5.0 \mu \mathrm{g} / \mathrm{mL}$ of ETH, one isolate with discordant results was to be false resistance, giving a sensitivity of $75 \%$. This strain harbored an S266R of ethA mutation related to ETH resistance (Table 4). Based on the suggested concentration for ETH, MGIT960 test results compare poorly with those of the L-J method, at $86.2 \%$ concordance. Although the $86.2 \%$ agreement was found, this population with resistant is small. This discordance is not a novel observation, although its extent is larger than that observed in previous studies $[12,28]$. Acute ETH DST has always been difficult to obtain because the drug is thermolabile [29]. ETH is an important drug for the treatment of MDR-TB and required for further DST testing and studies.

For PAS, an MIC of $4.0 \mu \mathrm{g} / \mathrm{mL}$ was reported [19]. PAS was tested among $0.5 \sim 16.0 \mu \mathrm{g} / \mathrm{mL}$ in this study (Table 2 ).

PAS, when applying the $2.0 \mu \mathrm{g} / \mathrm{mL}$ for the MGIT method, was $91 \%$ concordant ( $94.1 \%$ sensitivity, $87.5 \%$ specificity). One discrepant strain from new cases patients had low-level resistance (at $0.5 \mu \mathrm{g} / \mathrm{mL}$ ) but susceptible at $>1.0 \mu \mathrm{g} / \mathrm{mL}$.

The agreement for ETH and PAS was low, since we were not able to decide the concentration at which $100 \%$ of the resistant strains grew in the presence of the drug and $100 \%$ of the susceptible strains were inhibited. Larger studies are needed to define the further susceptibility test. 
In summary, MGIT960 and L-J proportion methods for second-line DST for M. tuberculosis were established for six drugs. The critical concentration for moxifloxacin was determined $0.5 \mu \mathrm{g} / \mathrm{mL}$, for levofloxacin $1.0 \mu \mathrm{g} / \mathrm{mL}$, for kanamycin $2.5 \mu \mathrm{g} / \mathrm{mL}$, for capreomycin $2.5 \mu \mathrm{g} / \mathrm{mL}$, for ethionamide $5.0 \mu \mathrm{g} / \mathrm{mL}$, and $\rho$-aminosalicylic acid $2.0 \mu \mathrm{g} / \mathrm{mL}$ using MGIT 960 . These critical concentrations were reliable for testing 2 nd line drug resistance. The two drugs whose ETH and PAS were low with agreement are required for further DST testing and studies.

\section{Acknowledgments}

This study was supported by a research grant of the Korea Centers of Disease Control and Prevention (CDC), Republic of Korea (Grant no. 2009-E00479-00). Special thanks to Mr. Byungchul Ahn, Jangho Park, and Ms. Heekyung Yu for the preparation of the materials.

\section{References}

[1] M. A. Aziz, A. Wright, A. Laszlo et al., "Epidemiology of antituberculosis drug resistance (the Global Project on Antituberculosis Drug Resistance Surveillance): an updated analysis," Lancet, vol. 368, no. 9553, pp. 2142-2154, 2006.

[2] R. Banerjee, G. F. Schecter, J. Flood, and T. C. Porco, "Extensively drug-resistant tuberculosis: new strains, new challenges," Expert Review of Anti-Infective Therapy, vol. 6, no. 5, pp. 713724, 2008.

[3] N. R. Gandhi, A. Moll, A. W. Sturm et al., "Extensively drugresistant tuberculosis as a cause of death in patients co-infected with tuberculosis and HIV in a rural area of South Africa," Lancet, vol. 368, no. 9547, pp. 1575-1580, 2006.

[4] M. C. Raviglione and I. M. Smith, "XDR tuberculosis-implications for Global Public Health," New England Journal of Medicine, vol. 356, no. 7, pp. 656-659, 2007.

[5] N. S. Shah, A. Wright, G. H. Bai et al., "Worldwide emergence of extensively drug-resistant tuberculosis," Emerging Infectious Diseases, vol. 13, no. 3, pp. 380-387, 2007.

[6] A. Martin, J. C. Palomino, and F. Portaels, "Rapid detection of ofloxacin resistance in Mycobacterium tuberculosis by two low-cost colorimetric methods: resazurin and nitrate reductase assays," Journal of Clinical Microbiology, vol. 43, no. 4, pp. 16121616, 2005.

[7] N. Morcillo, B. Di Giulio, B. Testani, M. Pontino, C. Chirico, and A. Dolmann, "A microplate indicator-based method for determining the susceptibility of multidrug-resistant Mycobacterium tuberculosis to antimicrobial agents," International Journal of Tuberculosis and Lung Disease, vol. 8, no. 2, pp. 253-259, 2004.

[8] T. Prammananan, W. Arjratanakool, A. Chaiprasert et al., "Second-line drug susceptibilities of Thai multidrug-resistant Mycobacterium tuberculosis isolates," International Journal of Tuberculosis and Lung Disease, vol. 9, no. 2, pp. 216-219, 2005.

[9] P. Bemer, T. Bodmer, J. Munzinger, M. Perrin, V. Vincent, and H. Drugeon, "Multicenter evaluation of the MB/BACT system for susceptibility Testing of Mycobacterium tuberculosis," Journal of Clinical Microbiology, vol. 42, no. 3, pp. 1030-1034, 2004.

[10] L. Heifets, T. Linder, T. Sanchez, D. Spencer, and J. Brennan, "Two liquid medium systems, mycobacteria growth indicator tube and MB redox tube, for Mycobacterium tuberculosis isolation from sputum specimens," Journal of Clinical Microbiology, vol. 38, no. 3, pp. 1227-1230, 2000.

[11] I. Bastian, L. Rigouts, J. C. Palomino, and F. Portaels, "Kanamycin susceptibility testing of Mycobacterium tuberculosis using Mycobacterium growth indicator tube and a colorimetric method," Antimicrobial Agents and Chemotherapy, vol. 45, no. 6, pp. 1934-1936, 2001.

[12] A. Krüüner, M. D. Yates, and F. A. Drobniewski, "Evaluation of MGIT 960-based antimicrobial testing and determination of critical concentrations of first- and second-line antimicrobial drugs with drug-resistant clinical strains of Mycobacterium tuberculosis," Journal of Clinical Microbiology, vol. 44, no. 3, pp. 811-818, 2006.

[13] M. Palaci, S. Y. M. Ueki, D. N. Sato, M. Alice da Silva Telles, M. Curcio, and E. A. M. Silva, "Evaluation of mycobacteria growth indicator tube for recovery and drug susceptibility testing of Mycobacterium tuberculosis isolates from respiratory specimens," Journal of Clinical Microbiology, vol. 34, no. 3, pp. 762764, 1996.

[14] S. Rüsch-Gerdes, G. E. Pfyffer, M. Casal, M. Chadwick, and S. Siddiqi, "Multicenter laboratory validation of the BACTEC MGIT 960 technique for testing susceptibilities of Mycobacterium tuberculosis to classical second-line drugs and newer antimicrobials," Journal of Clinical Microbiology, vol. 44, no. 3, pp. 688-692, 2006.

[15] M. Sharma, L. Thibert, P. Chedore et al., "Canadian multicenter laboratory study for standardized second-line antimicrobial susceptibility testing of Mycobacterium tuberculosis," Journal of Clinical Microbiology, vol. 49, no. 12, pp. 4112-4116, 2011.

[16] World Health Organization, "Policy guidance on drug-susceptibility testing (DST) of 2nd antituberculosis drug," WHO/ HTM/TB/2008.392. Geneva, Switzerland, 2008.

[17] K. M. Kam, A. Sloutsky, C. W. Yip et al., "Determination of critical concentrations of second-line anti-tuberculosis drugs with clinical and microbiological relevance," International Journal of Tuberculosis and Lung Disease, vol. 14, no. 3, pp. 282-288, 2010.

[18] G. Canetti, W. Fox, A. Khomenko et al., "Advances in techniques of testing mycobacterial drug sensitivity, and the use of sensitivity tests in tuberculosis control programmes," Bulletin of the World Health Organization, vol. 41, no. 1, pp. 21-43, 1969.

[19] C. Rodrigues, J. Jani, S. Shenai, P. Thakkar, S. Siddiqi, and A. Mehta, "Drug susceptibility testing of Mycobacterium tuberculosis against second-line drugs using the Bactec MGIT 960 System," International Journal of Tuberculosis and Lung Disease, vol. 12, no. 12, pp. 1449-1455, 2008.

[20] J. Van Ingen, S. Simons, R. De Zwaan et al., "Comparative study on genotypic and phenotypic second-line drug resistance testing of Mycobacterium tuberculosis complex isolates," Journal of Clinical Microbiology, vol. 48, no. 8, pp. 2749-2753, 2010.

[21] C. Piersimoni, C. Lacchini, V. Penati et al., "Validation of the agar proportion and 2 liquid systems for testing the susceptibility of Mycobacterium tuberculosis to moxifloxacin," Diagnostic Microbiology and Infectious Disease, vol. 57, no. 3, pp. 283-287, 2007.

[22] S. Y. G. Lin, E. Desmond, D. Bonato, W. Gross, and S. Siddiqi, "Multicenter evaluation of Bactec MGIT 960 system for secondline drug susceptibility testing of Mycobacterium tuberculosis complex," Journal of Clinical Microbiology, vol. 47, no. 11, pp. 3630-3634, 2009.

[23] C. A. Sanders, R. R. Nieda, and E. P. Desmond, "Validation of the use of middlebrook 7H10 agar, BACTEC MGIT 960, and 
BACTEC 460 12B media for testing the susceptibility of Mycobacterium tuberculosis to levofloxacin," Journal of Clinical Microbiology, vol. 42, no. 11, pp. 5225-5228, 2004.

[24] F. Giannoni, E. Iona, F. Sementilli et al., "Evaluation of a new line probe assay for rapid identification of gyrA mutations in Mycobacterium tuberculosis," Antimicrobial Agents and Chemotherapy, vol. 49, no. 7, pp. 2928-2933, 2005.

[25] T. S. Huang, S. S. J. Lee, H. Z. Tu et al., "Use of MGIT 960 for rapid quantitative measurement of the susceptibility of Mycobacterium tuberculosis complex to ciprofloxacin and ethionamide," Journal of Antimicrobial Chemotherapy, vol. 53, no. 4, pp. 600-603, 2004.

[26] S. Feuerriegel, H. S. Cox, N. Zarkua et al., "Sequence analyses of just four genes to detect extensively drug-resistant Mycobacterium tuberculosis strains in multidrug-resistant tuberculosis patients undergoing treatment," Antimicrobial Agents and Chemotherapy, vol. 53, no. 8, pp. 3353-3356, 2009.

[27] B. Springer, K. Lucke, R. Calligaris-Maibach, C. Ritter, and E. C. Böttger, "Quantitative drug susceptibility testing of Mycobacterium tuberculosis by use of MGIT 960 and EpiCenter instrumentation," Journal of Clinical Microbiology, vol. 47, no. 6, pp. 1773-1780, 2009.

[28] M. J. Ruiz-Serrano, L. Alcala, L. Martinez et al., "In vitro activities of six fluoroquinolones against 250 clinical isolates of Mycobacterium tuberculosis susceptible or resistant to first-line antituberculosis drugs," Antimicrobial Agents and Chemotherapy, vol. 44, no. 9, pp. 2567-2568, 2000.

[29] D. A. Mitchison, "Drug resistance in tuberculosis," European Respiratory Journal, vol. 25, no. 2, pp. 376-379, 2005. 


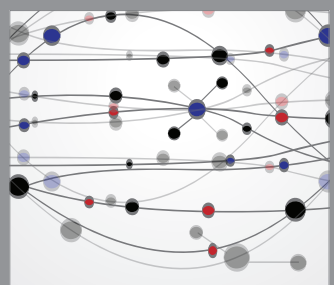

The Scientific World Journal
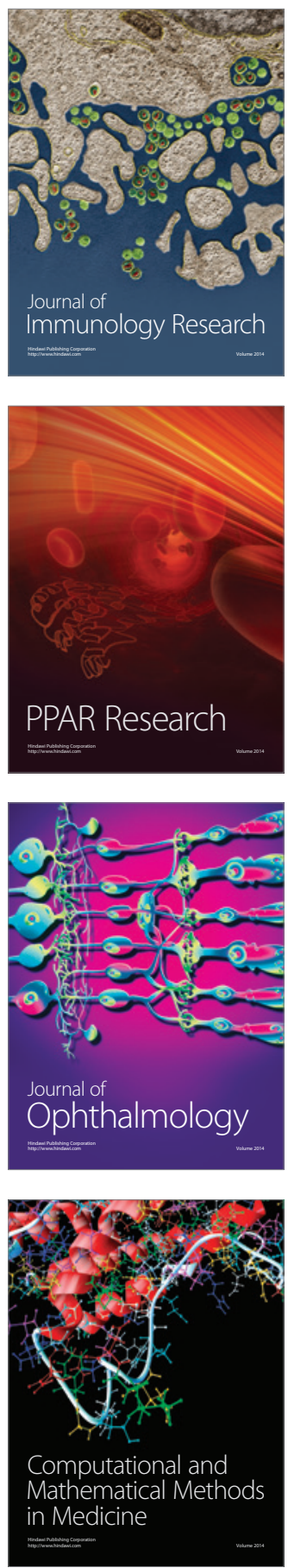

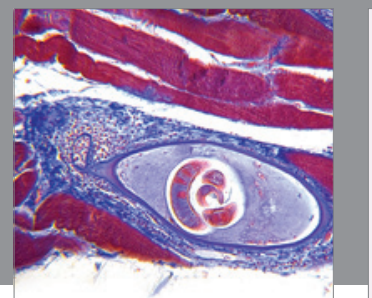

Gastroenterology

Research and Practice
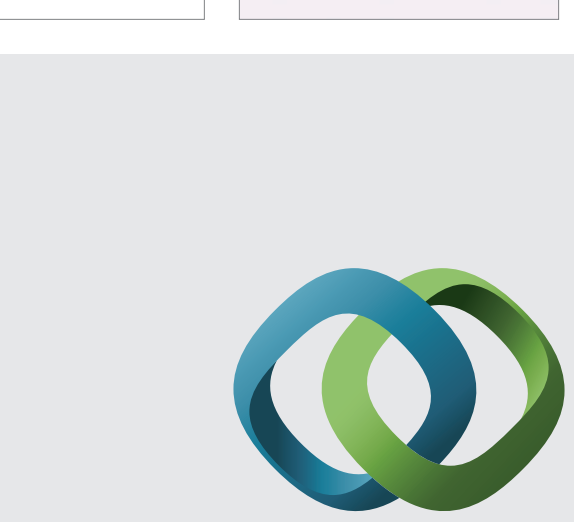

\section{Hindawi}

Submit your manuscripts at

http://www.hindawi.com
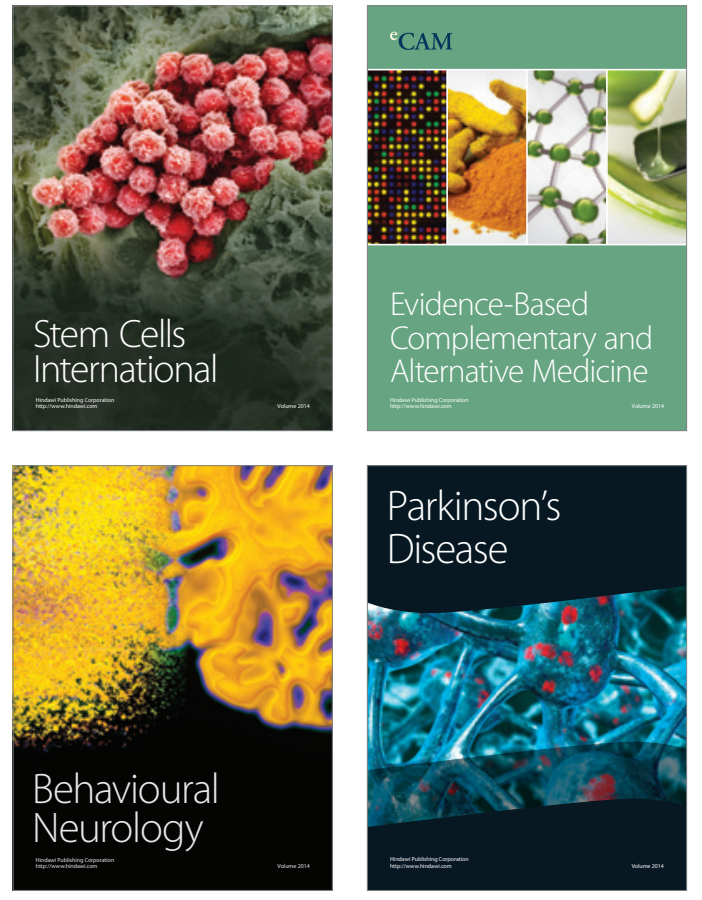
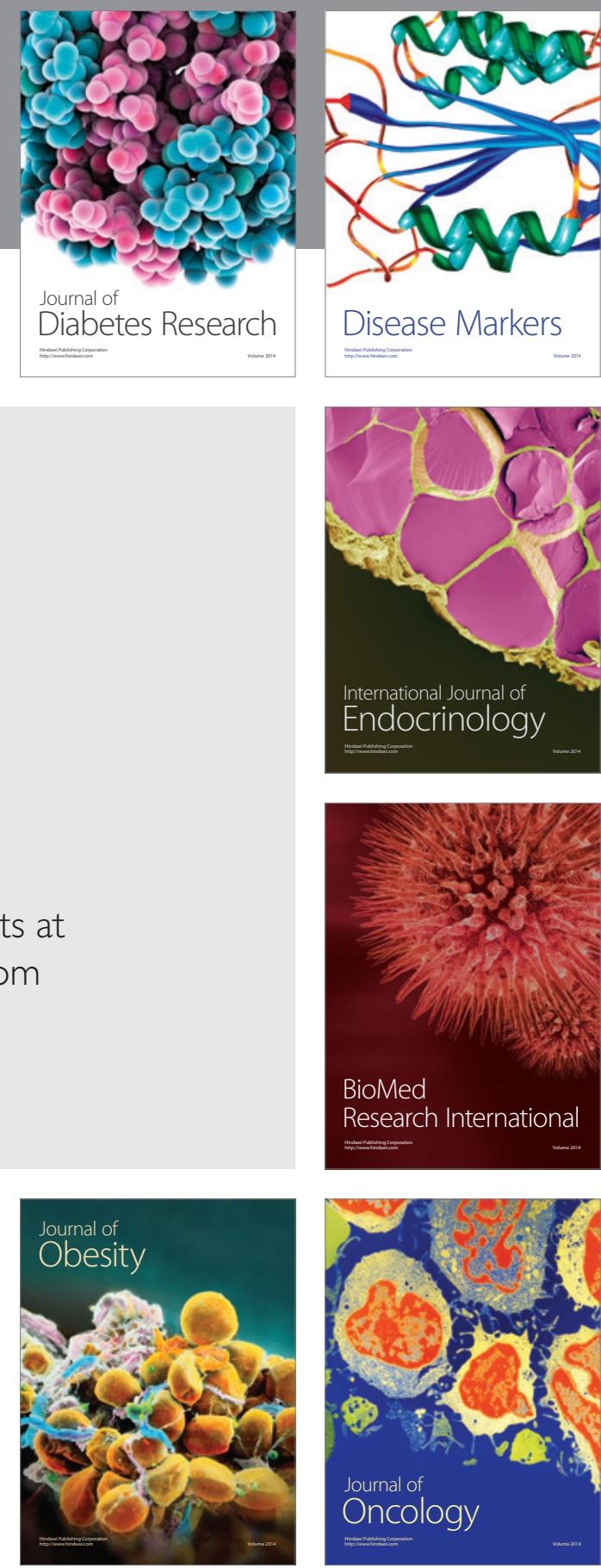

Disease Markers
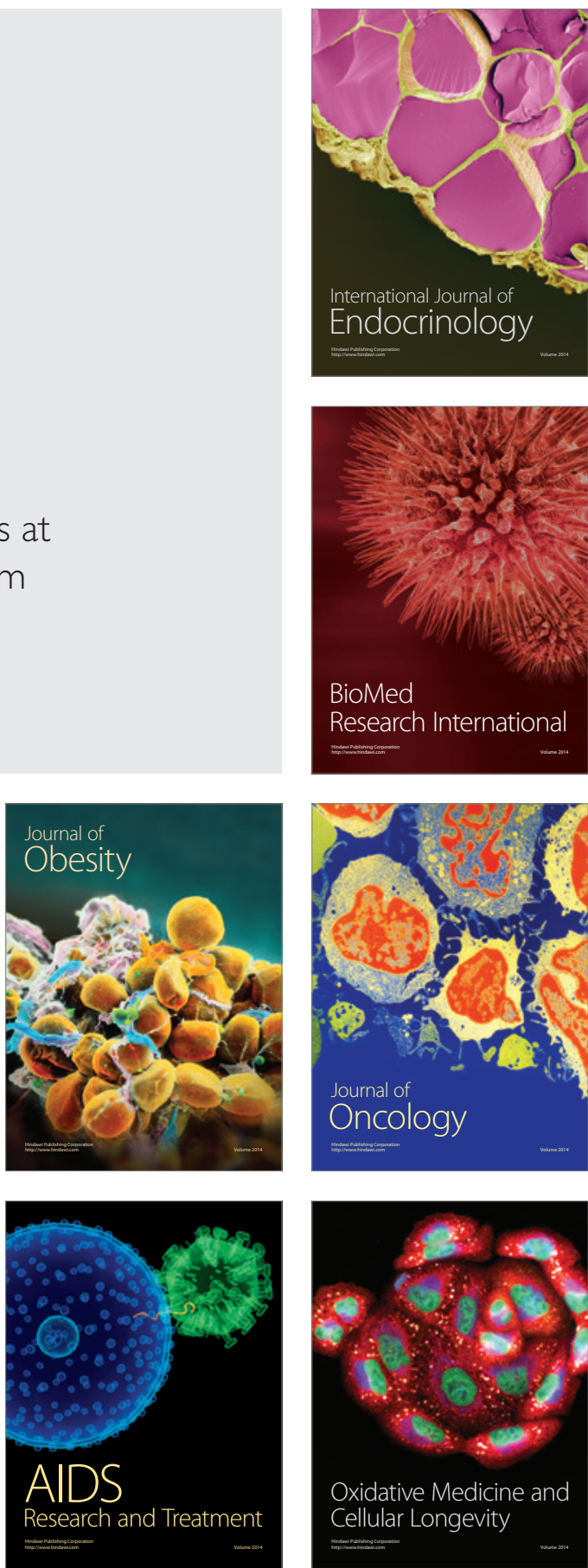